\title{
Mutation Fixation
}

National Cancer Institute

\section{Source}

National Cancer Institute. Mutation Fixation. NCI Thesaurus. Code C19682.

In avoiding recognition and removal by the mismatch repair system, a Mutation Fixation involves stabilization of a DNA sequence alteration in both strands so that the sequence change is propagated to progeny cells. 